\title{
On the Low-Frequency Natural Response of Conducting and Permeable Targets
}

\author{
Norbert Geng, Member, IEEE, Carl E. Baum, Fellow, IEEE, and Lawrence Carin, Senior Member, IEEE
}

\begin{abstract}
The low-frequency natural response of conducting, permeable targets is investigated. We demonstrate that the source-free response is characterized by a sum of nearly purely damped exponentials, with the damping constants strongly dependent on the target shape, conductivity, and permeability, thereby representing a potential tool for pulsed electromagnetic induction (EMI) identification (discrimination) of conducting and permeable targets. This general concept is then specialized to the particular case of a body of revolution (BOR), for which the Method-of-Moments (MoM)-computed natural damping constants from several targets are compared with measurements. Moreover, theoretical natural (equivalent) surface currents and damping coefficients are shown for other targets of interest. Finally, we investigate the practical use of such natural signatures in the context of identification, wherein Cramer-Rao bound (CRB) studies address signal-to-noise ratio (SNR) considerations.
\end{abstract}

\section{INTRODUCTION}

$\mathbf{T}$ HE NATURAL (source-free) modes of a linear system are independent of the driving function inducing their excitation [1]-[6] and therefore constitute useful signatures for identification (however, the excitation strengths of the various modes are generally strongly dependent on the incident fields). In electromagnetics, interest in such natural modes motivated development of the singularity expansion method (SEM) [7]-[15], which demonstrated that multiple interactions between scattering centers-after the incident fields have interrogated the entire target (at what are termed "late times")—can be repackaged compactly in terms of a sum of oscillatory, exponentially damped resonances (i.e., in terms of the SEM resonant modes). While the amplitudes of the SEM modes are dependent on the excitation waveform, the complex resonant frequencies themselves are termed "aspect independent" (the same for all target orientations and incident fields). After the original SEM framework was formalized [13], numerous researchers applied it to modeling [7]-[8], measurements [9]-[10], and signal processing (termed electromagnetic singularity identification, EMSI) [11]-[12], motivated largely by its potential for aspect-independent target identification.

The resonant frequencies of a particular target are determined by the spatial separation between scattering centers (i.e., by characteristic target lengths). For example, considering a conducting wire of length $d$, we would expect resonant

Manuscript received July 21, 1997; revised March 16, 1998.

N. Geng and L. Carin are with the Department of Electrical and Computer Engineering, Duke University, Durham, NC 27708-0291 USA (e-mail: lcarin@ee.duke.edu).

C. E. Baum is with Phillips Laboratory/WSQW, Kirtland Air Force Base, Albuquerque, NM 87117-5776 USA.

Publisher Item Identifier S 0196-2892(99)00119-9. frequencies at approximately $f_{n}=c n /(2 d)$, where $c$ is the speed of light and $n>0$ is an integer. However, if we excite a target at frequencies below its lowest SEM resonance, the fields and currents on the target will still exist after departure of the incident fields, except the late-time fields will not oscillate in the manner characteristic of resonances. Considering excitation of the wire at frequencies $f \ll f_{1}$, we would expect the late-time fields to decay approximately as $\exp [-(R / L) t]$, where $R$ and $L$ are the wire resistance and inductance, respectively (the inductance can be readily computed if we consider a wire loop [3]). In the former, resonant regime, the late-time fields are characterized by oscillations at nearly real frequencies (oscillatory naturalmode poles near the real-frequency axis), while the latter case is characterized approximately by exponential damping (evanescent natural-mode poles near the imaginary-frequency axis). These two extremes are characteristic as well of the modes of a resonant cavity, at frequencies above and below the lowest cavity resonance, respectively.

In this paper, we demonstrate that the low-frequency exponential damping characteristic of the simple wire example above can be extended to general target shapes and materials. This is done by employing 1) low-frequency circuit analogies (analogous to the wire example above) as well as through 2) development of a full-wave Method-of-Moments (MoM) analysis, with particular application to a conducting, permeable body of revolution (BOR). For such targets, it is demonstrated that the damping is strongly dependent on the target shape and material parameters. Therefore, if we can measure such exponentially damped waveforms accurately, the damping coefficient can be used for possible target identification. Of particular interest here are frequencies less than $1 \mathrm{MHz}$, for which the conductivity- and permeability-dependent skin depth can vary significantly (manifested in wider decayconstant variability with target properties, thereby aiding in discrimination). This should be contrasted with microwave and millimeter-wave frequencies, for which most metals can be considered perfect conductors (and therefore only the shape of the metal target influences its scattering properties).

An additional benefit of the low-frequency, pulsed-decay discrimination scheme discussed above is that, for the good conductors and low frequencies of interest here, the conduction current induced in most good metals is often considerably stronger than the displacement current, which can therefore often be neglected. Thus, the dielectric properties of the surrounding media are often relatively unimportant. This is particularly important for the low-frequency (termed elec- 
tromagnetic induction, EMI) identification of buried targets (e.g., mines), for example, for which the soil properties can often be ignored since the conductivity of the soil is generally many orders of magnitude less than that of good metals. Analogous to the EMSI referred above, we term this magnetic singularity identification (MSI). By contrast, groundpenetrating radar (GPR) [16]-[20], and the associated EMSI, is strongly influenced and limited by the soil properties. As a caveat, however, we should note that, if the metal target is very small, the conduction currents induced in the expansive, lowloss soil may become comparable to or exceed the currents induced in a small, localized, high-conductivity target, at which point the background medium does become important (this is particularly relevant for low-metal-content mines, for example); this will be the subject of future research.

We restrict ourselves here to the special case of conducting, permeable targets in free space (as mentioned, the soil conductivity and permittivity not being significant at the relevant low frequencies [3]). After using circuit theory to explain the basic wave phenomenology, numerical damping coefficients and currents are computed for the special case of conducting, permeable BOR's. While such analyses have been applied to the case of scattering from perfectly conducting and lowloss dielectric BOR's [21]-[24], [25]-[30], its application to calculation of natural modes for highly (but not perfectly) conducting, permeable targets is new. To validate the accuracy of the numerical data, comparisons are made with measured data [31]-[32] for several variations in material properties. Additionally, we present numerical results for the decay coefficients and natural-mode currents of other conducting and permeable targets.

Finally, in the context of target identification, such decay constants must be extracted from measured data, which are generally noisy. For such situations, a model-based estimation algorithm is often applied [14]-[15]. Each noisy measured waveform constitutes one realization of a random process, and therefore the estimated decay coefficients are random variables. Assuming the estimation algorithm is unbiased (the estimates for the decay constants are correct in the mean), we are interested in their variance, from which we quantify the expected accuracy of the decay-constant estimates for a given measured waveform. In this context, we investigate the Cramer-Rao bound (CRB) [33]-[35] for estimation of the damping coefficient of a noisy, exponentially damped waveform. The CRB constitutes the lowest variance any unbiased estimator can achieve for such parameter estimation. The CRB is strongly dependent on the signal-to-noise ratio (SNR). Therefore, by employing the CRB, we quantify the SNR requirements for unambiguous (low-variance) estimation of pulsed-EMI decay constants. Such an analysis represents an example in which wave modeling and basic signal processing coalesce to yield fundamental system constraints (SNR requirements in this case).

The remainder of the text is organized as follows. In Section II, we present a circuits-based explanation of the damped low-frequency EMI response from conducting, permeable targets, from which fundamental relationships are derived. We also formulate an appropriate boundary integral equation for the special case of conducting, permeable BOR's, which we solve via the MoM. The latter numerical formulation is discussed only briefly since similar analyses have been performed previously for different but related problems. In Section III, we address the issue of extracting exponentially damped waveforms from noisy measured data, through consideration of the CRB. The results of the numerical code are compared with measurements in Section IV, and several additional sets of numerical results are presented as well. Finally, conclusions are addressed in Section V.

\section{THEORY}

\section{A. Circuit-Based Explanation of Damped Low-Frequency EMI Response}

We first consider an approximate circuit-based analysis of low-frequency scattering from conducting targets. This simple analysis demonstrates the basic phenomenology of low-frequency, time-domain scattering from such targets, motivating the rigorous MoM analysis discussed subsequently.

An incident low-frequency magnetic field $\boldsymbol{H}_{\text {inc }}$ induces currents $\boldsymbol{J}\left(\boldsymbol{r}^{\prime}\right)$ in a conducting object. The magnetic dipole moment

$$
\boldsymbol{m}=\frac{1}{2} \iiint_{V^{\prime}}\left[\boldsymbol{r}^{\prime} \times \boldsymbol{J}\left(\boldsymbol{r}^{\prime}\right)\right] d V^{\prime}
$$

associated with these currents generates the scattered field $H^{\text {scat }}$. For observation distances large relative to characteristic target dimensions, the scattered quasimagnetostatic field can be expressed as [3], [6]

$$
\begin{aligned}
\boldsymbol{H}^{\text {scat }}(\boldsymbol{r}) & =\frac{1}{4 \pi r^{3}}[3 \hat{\boldsymbol{r}} \hat{\boldsymbol{r}}-\stackrel{\leftrightarrow}{\boldsymbol{I}}] \cdot \boldsymbol{m} \\
& =\frac{1}{4 \pi r^{3}}[3 \hat{\boldsymbol{r}} \hat{\boldsymbol{r}}-\overleftrightarrow{\boldsymbol{I}}] \cdot \overleftrightarrow{\boldsymbol{M}} \cdot \boldsymbol{H}^{\mathrm{inc}}(\boldsymbol{r}=\mathbf{0})
\end{aligned}
$$

where $\overleftrightarrow{I}$ is the identity dyadic and $\overleftrightarrow{M}$ is the magnetic polarizability dyadic, which relates the incident magnetic field to the induced magnetic dipole moment.

Let the scattering object have a simple shape so that it can have a magnetic moment in only a single direction, e.g., thin conducting disks or loops that support induced currents only in circular paths around the axis of symmetry (the magnetic moment of such an object is parallel to this axis, defined here as $z$ ). For simplification and because natural frequencies are independent of the excitation and observation point, we only consider a homogeneous $z$-directed incident magnetic field and the scattered field on the $z$-axis, expressed as

$$
H_{z}^{\text {scat }}(z)=\frac{m_{z}}{2 \pi r^{3}}=\frac{1}{2 \pi r^{3}} M_{z z} H_{z}^{\text {inc }}=\frac{1}{2 \pi r^{3}} M_{z z} H_{z 0} \text {. }
$$

Note that, if these assumptions are violated, only the amplitudes of the various modes will change, when the target is excited by a particular incident field.

The most simple target, a thin wire loop of area $A$, is characterized entirely by its resistance $R$ and inductance $L$, assuming that displacement currents can be neglected compared to the conduction currents (zero capacitance). By 
Faraday's law, an incident magnetic field parallel to the loop axis induces a voltage $V(t)=-\mu_{0} A \partial H_{z 0} / \partial t$ around the loop, which produces a current according to the impedance given by $R$ and $L$. Using the Laplace-transform variable $s=j \omega$ ( $\omega$ complex $)$, the loop current in the Laplace domain is given by [3], [19], [32]

$$
\begin{aligned}
I(s) & =\frac{V(s)}{Z(s)}=-\mu_{0} A \frac{s}{R+s L} H_{z 0}(s) \\
& =\frac{-\mu_{0} A}{L} \frac{s}{s+R / L} H_{z 0}(s)
\end{aligned}
$$

where we have assumed that the imposed field is initially zero. The current defines a magnetic moment $\boldsymbol{m}=I A \hat{z}$, and therefore, the $z$-directed scattered magnetic field can be written using (3) as

$$
H_{z}^{\text {scat }}(z, s)=\frac{-\mu_{0} A^{2}}{2 \pi r^{3} L} \frac{s}{s+R / L} H_{z 0}(s)
$$

resulting in a purely exponentially damped step response (i.e., for an excitation of the form $\left.H_{z 0}(t)=H_{z 0} u(t)\right)$ in the time domain

$$
\begin{aligned}
H_{z}^{\text {scat }}(z, t) & =\frac{-\mu_{0} A^{2}}{2 \pi r^{3} L} H_{z 0} e^{-t} u(t) \\
& =\frac{-\mu_{0} A^{2}}{2 \pi r^{3} L} H_{z 0} e^{-t / \tau_{\text {wirc }}} u(t)
\end{aligned}
$$

where $u(t)$ is the unit step function.

Next, the effects of the mutual coupling of multiple filament loops within a solid target are illustrated considering the simple case of two circular coaxial wire loops. The impedance matrix of the coupled system of the two loops may be written as

$$
[Z(s)]=\left[\begin{array}{ll}
Z_{11}(s) & Z_{12}(s) \\
Z_{21}(s) & Z_{22}(s)
\end{array}\right]=\left[\begin{array}{cc}
R_{1}+s L_{1} & s M \\
s M & R_{2}+s L_{2}
\end{array}\right]
$$

where $M$ stands for the mutual inductance of the two loops. A voltage is induced onto each loop from the incident magnetic field, which is again assumed to be homogeneous and parallel to the loop axes. The currents in the two loops are then

$$
\begin{aligned}
\left(\begin{array}{l}
I_{1}(s) \\
I_{2}(s)
\end{array}\right)= & {[Z(s)]^{-1}\left(\begin{array}{l}
V_{1}(s) \\
V_{2}(s)
\end{array}\right) } \\
= & \frac{-\mu_{0} s H_{z 0}(s)}{\left(R_{1}+s L_{1}\right)\left(R_{2}+s L_{2}\right)-(s M)^{2}} \\
& \cdot\left(\begin{array}{c}
\left(R_{2}+s L_{2}\right) A_{1}-s M A_{2} \\
-s M A_{1}+\left(R_{1}+s L_{1}\right) A_{2}
\end{array}\right)
\end{aligned}
$$

and therefore using (3) the scattered magnetic field (on the $z$-axis) can be written as

$$
\begin{aligned}
H_{z}^{\text {scat }}(z, s) & \\
= & \frac{A_{1} I_{1}(s)}{2 \pi r_{1}^{3}}+\frac{A_{2} I_{2}(s)}{2 \pi r_{2}^{3}} \\
\approx & \frac{1}{2 \pi r^{3}}\left[A_{1} I_{1}(s)+A_{2} I_{2}(s)\right] \\
\approx & \frac{-\mu_{0}}{2 \pi r^{3}} \frac{s\left(R_{2}+s L_{2}\right) A_{1}^{2}-2 s^{2} M A_{1} A_{2}+s\left(R_{1}+s L_{1}\right) A_{2}^{2}}{\left(R_{1}+s L_{1}\right)\left(R_{2}+s L_{2}\right)-(s M)^{2}} \\
& \cdot H_{z 0}(s)
\end{aligned}
$$

where we assumed an observation distance much larger than the loop separation. The resonances are given by the singularities of (9). If we write $s_{01}=-R_{1} / L_{1}=-1 / \tau_{\text {wire1 }}$ and $s_{02}=-R_{2} / L_{2}=-1 / \tau_{\text {wire2 }}$ for the negative real poles of each loop in the absence of the other loop, and $0 \leq k^{2}=M^{2} / L_{1} L_{2} \leq 1$ for the mutual coupling coefficient, the resonances are

$$
\begin{aligned}
s_{1,2} & =\frac{\left(s_{01}+s_{02}\right)+\sqrt{\left(s_{01}-s_{02}\right)^{2}+4 k^{2} s_{01} s_{02}}}{2\left(1-k^{2}\right)} \\
& =\frac{\left(s_{01}+s_{02}\right) \pm \sqrt{\left(s_{01}+s_{02}\right)^{2}-4\left(1-k^{2}\right) s_{01} s_{02}}}{2\left(1-k^{2}\right)}
\end{aligned}
$$

Again the poles of the system are negative real, corresponding to pure exponential damping. As expected, the poles reduce to those of the isolated loops if the mutual coupling vanishes $\left(k^{2}=0\right)$. For nonzero coupling, the step response of the system has the form

$$
\begin{aligned}
& H_{z}^{\text {scat }}(z, t)= \\
& \begin{aligned}
\frac{-\mu_{0}}{2 \pi r^{3}} H_{z 0}[ & f_{1}\left(A_{1,2}, R_{1,2}, L_{1,2}, k\right) e^{-t / \tau_{1}} \\
& \left.+f_{2}\left(A_{1,2}, R_{1,2}, L_{1,2}, k\right) e^{-t / \tau_{2}}\right] u(t) .
\end{aligned}
\end{aligned}
$$

For zero coupling between the two loops, it reduces to a superposition of the two step responses for the individual loops according to (6)

$$
\begin{aligned}
& H_{z}^{\text {scat }}(z, t) \\
& \quad=\frac{-\mu_{0}}{2 \pi r^{3}} H_{z 0}\left[\frac{A_{1}^{2}}{L_{1}} e^{-t / \tau_{\text {wirc } 1}}+\frac{A_{2}^{2}}{L_{2}} e^{-t / \tau_{\text {wirc } 2}}\right] u(t) .
\end{aligned}
$$

Considering the $R$ and $L$ of simple nonpermeable shapes (loops), we can show that the time constants are in general proportional to the square of some characteristic object dimension times the conductivity. In the context of our rigorous MoM studies discussed below, we will demonstrate that such properties have been found to hold for more complicated targets than the simple loops for which the circuit theory is directly applicable. Finally, the variation of the poles or decay time constants as a function of the permeability is more complicated and therefore cannot be derived easily using the simple circuit-based theory. A solution for a conducting and permeable sphere is given in [3], [19], and [32], but there is no analytical method available for arbitrary three-dimensional (3-D) objects. This can only be accomplished using numerical methods like the MoM solution for BOR's described next.

\section{B. Surface Integral Equation and MoM}

We consider electromagnetic scattering from highly (but not perfectly) conducting and permeable targets situated in a homogeneous environment, e.g., free space. Moreover, we specialize the solution to the case of a BOR. Because the surface integral equation approach for treating problems involving perfectly conducting as well as low-loss dielectric BOR's has 


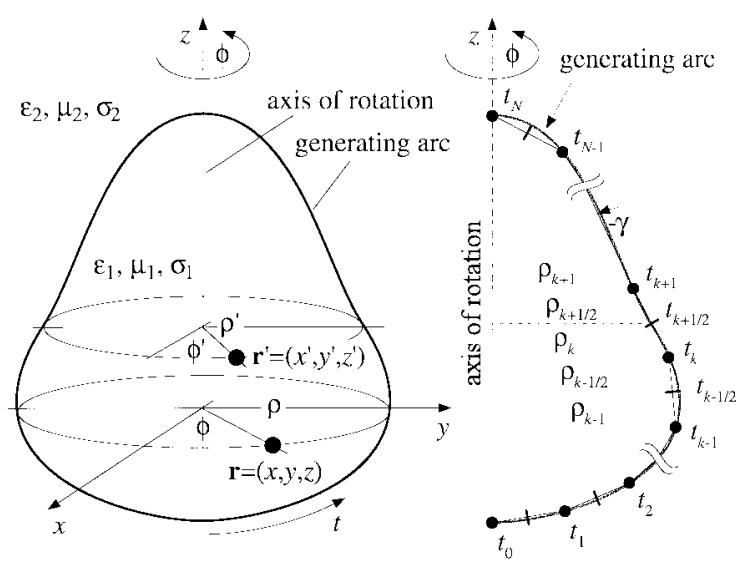

Fig. 1. General geometry for a body of revolution (BOR) and discretization scheme for MoM solution using a staggered pulse-basis along the generating arc [23], [24], [27].

been studied by various authors [21]-[23], [24]-[30], only a brief summary of the basic theory will be given here.

Considering Fig. 1, regions 1 and 2 are characterized by (possibly frequency dependent) medium parameters $\left(\varepsilon_{1}, \mu_{1}, \sigma_{1}\right)$ and $\left(\varepsilon, \mu_{2}, \sigma_{2}\right)$, respectively. According to surface equivalence principles [36]-[37], exterior scattered fields $\left(\boldsymbol{E}^{\text {scat }}, \boldsymbol{H}^{\text {scat }}\right)$ and interior total fields $(\boldsymbol{E}, \boldsymbol{H})$ may be determined from a set of equivalent electric and magnetic surface currents. These equivalent surface currents are related to the total tangential magnetic and electric field by $\boldsymbol{J}_{s}= \pm \hat{\boldsymbol{n}} \times \boldsymbol{H}$ and $\boldsymbol{M}_{s}= \pm \boldsymbol{E} \times \hat{\boldsymbol{n}}$, respectively, where the upper sign holds for the exterior and the lower sign for the interior region. Coupled integral equations

$$
\begin{aligned}
\boldsymbol{E}_{\mathrm{tan}}^{\mathrm{inc}}(\boldsymbol{r})= & {\left[\sum_{i=1}^{2}\left(j \omega \boldsymbol{A}_{i}(\boldsymbol{r})+\nabla \Phi_{e i}(\boldsymbol{r})\right)\right]_{\tan } } \\
& +\left[\nabla \times \sum_{i=1}^{2} \frac{\boldsymbol{F}_{i}(\boldsymbol{r})}{\sigma_{i}+j \omega \varepsilon_{i}}\right]_{\tan } \\
\boldsymbol{H}_{\mathrm{tan}}^{\mathrm{inc}}(\boldsymbol{r})= & {\left[-\nabla \times \sum_{i=1}^{2} \frac{A_{i}(\boldsymbol{r})}{\mu_{i}}\right]_{\tan } } \\
& +\left[\sum_{i=1}^{2}\left(j \omega \boldsymbol{F}_{i}(\boldsymbol{r})+\nabla \Phi_{m i}(\boldsymbol{r})\right)\right]_{\tan }
\end{aligned}
$$

for the surface currents are obtained by satisfying the boundary conditions at the interface [27]-[30], where $\left(\boldsymbol{E}_{\text {tan }}^{\text {inc }}, H_{\tan }^{\text {inc }}\right)$ represent the tangential components of the incident field. Herein the electric and magnetic vector and scalar potentials are defined as

$$
\begin{aligned}
\boldsymbol{A}_{i}(\boldsymbol{r}) & =\mu_{i} \oiint_{S^{\prime}} G_{i}\left(\boldsymbol{r}, \boldsymbol{r}^{\prime}\right) \boldsymbol{J}_{s}\left(\boldsymbol{r}^{\prime}\right) d S^{\prime} \\
\boldsymbol{F}_{i}(\boldsymbol{r}) & =\frac{\sigma_{i}+j \omega \varepsilon_{i}}{j \omega} \oiint_{S^{\prime}} G_{i}\left(\boldsymbol{r}, \boldsymbol{r}^{\prime}\right) \boldsymbol{M}_{s}\left(\boldsymbol{r}^{\prime}\right) d S^{\prime} \\
\Phi_{e i}(\boldsymbol{r}) & =\frac{j \omega}{\sigma_{i}+j \omega \varepsilon_{i}} \oiint_{S^{\prime}} G_{i}\left(\boldsymbol{r}, \boldsymbol{r}^{\prime}\right) q_{e s}\left(\boldsymbol{r}^{\prime}\right) d S^{\prime} \\
& =\frac{-1}{\sigma_{i}+j \omega \varepsilon_{i}} \oiint_{S^{\prime}} G_{i}\left(\boldsymbol{r}, \boldsymbol{r}^{\prime}\right) \nabla^{\prime} \cdot \boldsymbol{J}_{s}\left(\boldsymbol{r}^{\prime}\right) d S^{\prime}
\end{aligned}
$$

$$
\begin{aligned}
\Phi_{m i}(\boldsymbol{r}) & =\frac{1}{\mu_{i}} \oiint_{S^{\prime}} G_{i}\left(\boldsymbol{r}, \boldsymbol{r}^{\prime}\right) q_{m s}\left(\boldsymbol{r}^{\prime}\right) d S^{\prime} \\
& =\frac{-1}{j \omega \mu_{i}} \oiint_{S^{\prime}} G_{i}\left(\boldsymbol{r}, \boldsymbol{r}^{\prime}\right) \nabla^{\prime} \cdot \boldsymbol{M}_{s}\left(\boldsymbol{r}^{\prime}\right) d S^{\prime}
\end{aligned}
$$

with the Green's function

$$
\begin{aligned}
G_{i}\left(\boldsymbol{r}, \boldsymbol{r}^{\prime}\right) & =\frac{e^{-j k_{i}\left|\boldsymbol{r}-\boldsymbol{r}^{\prime}\right|}}{4 \pi\left|\boldsymbol{r}-\boldsymbol{r}^{\prime}\right|} \text { with } \\
k_{i} & =\sqrt{\omega^{2} \varepsilon_{i} \mu_{i}-j \omega \mu_{i} \sigma_{i}} \text { and } i=1,2
\end{aligned}
$$

for the homogeneous interior $(i=1)$ and homogeneous exterior $(i=2)$ region, respectively. The complex wavenumbers of the interior and exterior region are given by $k_{1}$ and $k_{2}$, and the vectors $\boldsymbol{r}$ and $\boldsymbol{r}^{\prime}$ represent source and observation point, respectively.

Equations (13)-(16) are valid for an arbitrarily shaped homogeneous target in a homogeneous environment, for which an MoM solution could be applied [36]. However, we only consider the special case of a BOR (Fig. 1), which is formed by rotating a generating arc about an axis that is chosen to be the $z$-axis of a Cartesian coordinate system. For numerical simulations, the generating arc is approximated by a sequence of linear segments. Coordinates $(\phi, t)$ are introduced, where the angle $\phi$ is equal to the one used in cylindrical coordinates $(\rho, \phi, z)$ and $t$ is the length variable along the curve generating the arc. To take advantage of the rotational symmetry, the incident field, all currents, and scalar Green's functions are expanded into discrete Fourier series by taking a Fourier transform in the azimuthal $(\phi)$ direction, from which the general solution reduces to an infinite number of distinct MoM problems for each of the Fourier modes (each with azimuthal variation $\exp (j m \phi))$. The resulting sets of simultaneous equations may be represented in matrix form as

$$
\left[Z^{m}\right] \boldsymbol{I}^{m}=\boldsymbol{V}^{m} \text { for } m=0, \pm 1, \pm 2, \pm 3, \cdots
$$

where $\left[Z^{m}\right]$ is the moment matrix, $\boldsymbol{I}^{m}$ is a column vector containing the unknown basis function coefficients, and $\boldsymbol{V}^{m}$ is the driving vector for the $m$ th Fourier mode [21]-[23], [25]-[26]. Details regarding the calculation of the impedance matrix or the driving vector can be found in the literature.

If we are interested in the natural resonances of the target, the driving vector $\boldsymbol{V}^{m}$ is set to zero. Then the system (17) of linear equations has a nontrivial solution only if the determinant of the MoM impedance matrix is zero

$$
\begin{aligned}
\operatorname{det}\left[Z^{m}\left(s_{m, \nu}=j \omega_{m, \nu}\right]\right. & =0 \quad \text { with } \\
s_{m, \nu} & =j \omega_{m, \nu}=j\left[\omega_{m, \nu}^{\prime}+j \omega_{m, \nu}^{\prime \prime}\right] \\
& =-\omega_{m, \nu}^{\prime \prime}+j \omega_{m, \nu}^{\prime} .
\end{aligned}
$$

The roots of (18) in the complex frequency plane are the resonant frequencies of the modes $(m, \nu)$. Searching for these complex roots is relatively easy because, for highly conducting and permeable targets, the negative real part of $s_{m, \nu}$ is much larger than the imaginary part (almost pure exponential damping, as expected from Section II-A). Thus, it is sufficient to search first along the negative real $s$ axis for an approximate solution, after which Mueller's method is performed to yield an 
accurate solution for the complex resonant frequency (where we have found five-ten iterative steps generally sufficient).

For mode identification, it is in principle necessary to compute the detailed field distribution inside and outside the target. But in a limited sense, this may be accomplished by studying the resonant surface current distribution only. For each complex resonant frequency, the surface currents or, equivalently, the tangential fields along the surface can be readily calculated.

In contrast to MoM calculations for perfectly conducting objects, for which subsectional basis-function discretization only depends on the wavelength in the outside region (often free space), the maximum subsection length here has to be chosen with respect to the wavenumber inside the highly conducting and permeable object. Neglecting displacement currents and assuming a real permeability $\mu_{1}$, this wavenumber is given by

$$
k_{1}=k_{1}^{\prime}-j k_{1}^{\prime \prime}=\sqrt{\frac{\sigma_{1} \mu_{1}}{2}}\left(\sqrt{|\omega|+\omega^{\prime \prime}}-j \sqrt{|\omega|-\omega^{\prime \prime}}\right) .
$$

Reasonable results are obtained if the subsection length $\Delta t$ (along the generating arc) satisfies the conditions $k_{1}^{\prime} \Delta t \leq 2 \pi / 10$ and $k_{1}^{\prime \prime} \Delta t \leq 1 / 2$, which means at least ten subsections per wavelength and at least two per skin depth. Although the required number of subdomains for a target that is much larger than the skin depth is impractical, this does not cause any problems because, for these cases (high frequency, high conductivity, and/or large target), the problem can be easily solved using a perfectly electric conducting (PEC) assumption.

Considering the wavenumber in (19), we discuss an alternative means of deriving the resonant frequency scaling relationship discussed in Section II-A (i.e., the relationship that the decay constant is proportional to the product of conductivity times the square of a characteristic target dimension). For purely imaginary frequencies, the wavenumber is real. Assuming real permeabilities $\mu_{1} \gg \mu_{2}$ and a highly conducting target $\left(\left|\sigma_{1}+j \omega \varepsilon_{1}\right| \approx \sigma_{1} \gg\left|\sigma_{2}+j \omega \varepsilon_{2}\right|\right)$, from the theory of cavities or dielectric resonators [37], [28]-[30], the product of the real resonant wavenumber and a characteristic dimension $D$ of the object does not change for a given shape. This results in

$$
D \sqrt{\omega_{m, \nu}^{\prime \prime} \sigma_{1} \mu_{1}}=\mathrm{const} \Rightarrow \omega_{m, \nu}^{\prime \prime}=\frac{1}{\tau_{m, \nu}} \propto \frac{1}{D^{2} \sigma_{1} \mu_{1}} .
$$

\section{CRAMER-RAo LOWER Bound}

The previous section demonstrated that the low-frequency transient response from a conducting target is characterized by exponential damping. Moreover, the decay constant is a function of the target shape, conductivity, and permeability. Therefore, in principle, it is possible to use the decay constants for target identification by estimating them from measured data [9], [14]-[15]. However, for noisy data, the extracted decay parameters must be characterized as random variables, with particular mean and variance. Assuming the mean is correct (constituting an unbiased estimate), it is of interest to quantify the variance, from which we can assess the practicality of achieving unambiguous target identification via decay-constant discrimination. To this end, we consider the Cramer-Rao lower bound (CRLB) [33]-[34], which represents the lowest possible variance for estimation of particular parameters from noisy data. The CRLB is a function of the SNR. Therefore, if we can quantify the maximum tolerable decay-parameter variance, for unambiguous identification (based on the range of decay constants anticipated), the CRLB will then quantify what SNR is required for this variance to be achieved, from which critical system requirements can be assessed. It should be noted that the CRLB does not suggest that an estimator exists that can achieve this minimum variance (termed an efficient estimator), only that no estimator can do better; however, several nearly efficient estimators are available for the estimation of exponential waveforms [14]-[15].

Let the vector $\boldsymbol{r}$ represent a discretized version of the measured time-domain waveform (of $N$ samples)

$$
\boldsymbol{r}=\left[\begin{array}{llll}
r_{0} & r_{1} & r_{2} & \cdots r_{N-1}
\end{array}\right]^{T}=\boldsymbol{g}(\boldsymbol{a})+\boldsymbol{n}
$$

where $\boldsymbol{g}(\boldsymbol{a})$ is the $N$-dimensional signal vector and $\boldsymbol{n}$ represents additive noise (here, assumed white Gaussian noise with variance $\sigma^{2}$ ). The signal $\boldsymbol{g}$ is assumed to be a function of the $P$-dimensional vector $\boldsymbol{a}$, which is to be estimated.

The CRLB states that the covariance matrix $[C]$ of the estimated vector satisfies [33]-[34]

$$
[\boldsymbol{C}]-\left[\boldsymbol{I}(\boldsymbol{a}]^{-1}=E\left\{(\hat{\boldsymbol{a}}-\boldsymbol{a})(\hat{\boldsymbol{a}}-\boldsymbol{a})^{T}\right\}-[\boldsymbol{I}(\boldsymbol{a})]^{-1} \geq 0\right.
$$

where $\geq 0$ means that the matrix is positive semidefinite and the Fisher information matrix [33]-[34] is expressed as

$$
\begin{aligned}
{[I(\boldsymbol{a})]_{i j} } & =-E\left\{\frac{\partial^{2} \ln p_{\boldsymbol{r} \mid \boldsymbol{a}}(\boldsymbol{r} \mid \boldsymbol{a})}{\partial a_{i} \partial a_{j}}\right\} \\
& =E \underset{\text { with } \quad i, j=1, \cdots, P .}{\left\{\frac{\partial \ln p_{\boldsymbol{r} \mid \boldsymbol{a}}(\boldsymbol{r} \mid \boldsymbol{a})}{\partial a_{i}} \cdot \frac{\partial \ln p_{\boldsymbol{r} \mid \boldsymbol{a}}(\boldsymbol{r} \mid \boldsymbol{a})}{\partial a_{j}}\right\}}
\end{aligned}
$$

Therefore, the lower bounds for the variances of the individual estimates $\left(\hat{a}_{p}(p=1,2, \cdots, P)\right.$ are given by the $p$ th diagonal element in the inverse of the Fisher matrix. The partial derivatives in (23) are evaluated at the true values, and the expectation is taken with respect to the conditional probability density function $p_{\boldsymbol{r} \mid \boldsymbol{a}}(\boldsymbol{r} \mid \boldsymbol{a})$.

We are interested in the parameters describing the natural response of a system. Assuming $M$ poles $s_{m}$ of single order, which is true for the conducting and permeable targets of interest here [3], [19], the system function in the Laplace domain and the corresponding impulse response are given by

$$
\begin{aligned}
H(s) & =\sum_{m=1}^{M} \frac{B_{m}}{s-s_{m}} \Leftrightarrow h(t)=\sum_{m=1}^{M} B_{m} e^{s_{m} t} u(t) \\
& =\sum_{m=1}^{M} b_{m} e^{j \beta_{m}} e^{j 2 \pi f_{m}^{\prime} t} e^{-2 \pi f_{m}^{\prime \prime} t} u(t)
\end{aligned}
$$

where the excitation coefficients for pairs of conjugate complex poles $s_{i}=s_{j}^{*}$ satisfy $B_{i}=B_{j}^{*}$. Therefore the components 
of the signal vector $\boldsymbol{g}(\boldsymbol{a})$ can be written as

$$
g_{n}(\boldsymbol{a})=\sum_{m=1}^{M} b_{m} e^{j \beta_{m}} e^{j 2 \pi f_{m}^{\prime} n \Delta t} e^{-2 \pi f_{m}^{\prime \prime} n \Delta t}
$$

with $n=0,1,2, \cdots, N-1$, where $\Delta t$ represents the time increment for the sampling and, hence, $N \Delta t$ the total observation time. The vector $\boldsymbol{a}$ of the $P=4 M$ unknown parameters (excluding the noise variance $\sigma^{2}$ ) is given by

$$
\begin{aligned}
\boldsymbol{a} & =\left[\begin{array}{llllll}
a_{1} & a_{2} & \cdots & a_{p}
\end{array}\right]^{T} \\
& =\left[\begin{array}{lllllll}
b_{1} & \cdots & b_{M} & \beta_{1} & \cdots & \beta_{M} & f_{1}^{\prime} \\
& \cdots & f_{M}^{\prime} & f_{1}^{\prime \prime} & \cdots & f_{M}^{\prime \prime}
\end{array}\right]^{T}
\end{aligned}
$$

and the likelihood function $p_{\boldsymbol{r} \mid \boldsymbol{a}}(\boldsymbol{r} \mid \boldsymbol{a})$ is [33]-[34]

$$
\begin{aligned}
p_{\boldsymbol{r} \mid \boldsymbol{a}}(\boldsymbol{r} \mid \boldsymbol{a}) & =p_{\boldsymbol{n}}(\boldsymbol{r}-\boldsymbol{s}(\boldsymbol{a})) \\
& =\frac{1}{\left(2 \pi \sigma^{2}\right)^{N / 2}} \exp \left[-\frac{(\boldsymbol{r}-g(\boldsymbol{a}))^{T} \cdot(\boldsymbol{r}-g(\boldsymbol{a}))}{2 \sigma^{2}}\right] .
\end{aligned}
$$

Using this probability density function, the signal vector $g$ in (25), and the parameter vector $\boldsymbol{a}$ in (26), the Fisher information matrix $[\boldsymbol{I}(\boldsymbol{a})]$, and the CRLB matrix $[\boldsymbol{C}]$ can be derived [35] for all $4 M+1$ unknowns, including the noise variance $\sigma^{2}$. No further simplifications are necessary here, but for the actual computation of the CRLB matrix, the excitation strengths of the individual damped exponentials have to be known.

A simple approximate solution for the CRLB as a function of the SNR that does not require any knowledge of the excitation strengths can be derived, assuming known noise variance $\sigma^{2}$ and excitation of only the resonant mode of interest. This is a "best-case" study that provides a "lower bound" for the CRLB. Taking into account only the exponential decay term associated with the lowest purely imaginary resonant frequency (fundamental mode), i.e., neglecting all other poles, the time series simplifies to

$$
\begin{aligned}
g_{n}\left(B, f_{\text {res }}^{\prime \prime}\right) & =B e^{-2 \pi f_{\text {rcc }}^{\prime \prime} n \Delta t} \\
& =B e^{-n \Delta t / \tau} \quad \text { with } \quad n=0,1, \cdots, N-1
\end{aligned}
$$

where we have renamed the amplitude and the resonant frequency for simplification. After some algebra, and assuming a sampling time much smaller and a total observation time much larger than the decay time constant associated with this lowest resonant frequency, i.e.,

$$
\begin{aligned}
\Delta t / \tau & =2 \pi f_{\text {res }}^{\prime \prime} \Delta t \ll 1 \text { and } \\
N \Delta t / \tau & =2 \pi f_{\text {res }}^{\prime \prime} N \Delta t \gg 1
\end{aligned}
$$

the CRLB for the normalized resonant frequency or decay time, respectively, simplifies to

$$
\operatorname{var}\left\{\frac{\hat{f}_{\text {res }}^{\prime \prime}}{f_{\text {res }}^{\prime \prime}}\right\}=\operatorname{var}\left\{\frac{\hat{\tau}}{\tau}\right\}=4 \sigma^{2} / \sum_{n=0}^{N-1} g_{n}^{2}=\frac{4}{\mathrm{SNR}} .
$$

As expected, the variance is reduced with increasing SNR. We have found that the error incurred by using (30) instead of the general CRLB in (22) is less than 5\%, as long as the sampling time and the number of sampling points satisfy $\Delta t / \tau<0.55$ and $N \Delta t / \tau>3$.

\section{RESULTS}

The MoM code for BOR-type targets is used to calculate natural complex frequencies and resonant surface currents for different shapes and material parameters. For a sphere, thin disk, and cylinder, these results are compared with measurements. In addition, numerical results for a more general BOR are presented. Only results for $m=0$ will be shown here, these rotationally symmetric modes often being most important in practice. Since from now on we assume free space for medium 2 , we drop the subscript 1 to indicate material parameters of the target.

\section{A. Sphere}

To illustrate the frequency dependence of the MoM impedance matrix determinant, Fig. 2 shows its magnitude along the imaginary frequency axis for a sphere of radius $a=1.27 \mathrm{~cm}$ and azimuthal mode index $m=0$. Although the resonances are not exactly located on the imaginary axis, such a plot gives a good idea as to the number and location of the resonances. The imaginary frequencies at which the minima of the magnitude occur are used as starting points for a Mueller method to search for the complex roots. Fig. 2 also shows a comparison between different material properties. The curves for nonpermeable targets $\left(\mu_{r}=1\right)$ with conductivities $\sigma=5 \cdot 10^{6} \mathrm{~S} / \mathrm{m}$ and $\sigma=20 \cdot 10^{6} \mathrm{~S} / \mathrm{m}$ lie on top of each other (note the scaling of the frequency axis by a factor of four), which is consistent with the resonant frequency scaling relationship (20). The curves for $\sigma=5 \cdot 10^{6} \mathrm{~S} / \mathrm{m}$ and permeabilities $\mu_{r}=1$ and $\mu_{r}=100$, respectively, also use different frequency axes, here scaled by a factor of $100 . \mathrm{TM}^{z}$ resonances with $m=0$, i.e., modes with the magnetic field only in the azimuth direction (and therefore not excited by an incident $z$-directed magnetic field), scale exactly according to (20). For $\mathrm{TE}^{z}$-resonances, this is only true if the permeability satisfies $\mu \gg \mu_{0}$. A simple explanation for this will be given in Section IV-C.

In [31]-[32], an impulse detector was used to measure the step response of certain canonical objects, in which the impulse response of the detector itself has been deconvolved from the measured data. Using a Helmholtz coil, the generated incident magnetic field was nearly uniform, as assumed in Section II-A. The scattered field was measured with a quadrupole receiver coil. Table I presents a direct comparison of the measured and MoM-computed decay times for a 1-in diameter sphere made of aluminum, brass, lead, and 304 stainless steel, respectively [32]. Taking into account that in the literature the conductivities given for different metals are highly variable, the agreement between measurement and numerical results is excellent. In fact, assuming that the measurements were performed with a high degree of accuracy, we can use our theory to extract from data the exact conductivities for the targets considered in the experiments.

\section{B. Thin Disk}

Next we consider aluminum disks of diameter $2 a=5 \mathrm{~cm}$ and nominal thickness $3.175,1.588,0.794$, and 0.508 , respectively $(1 / 8,1 / 16,1 / 32$, and $1 / 50$ in), for which measured decay 


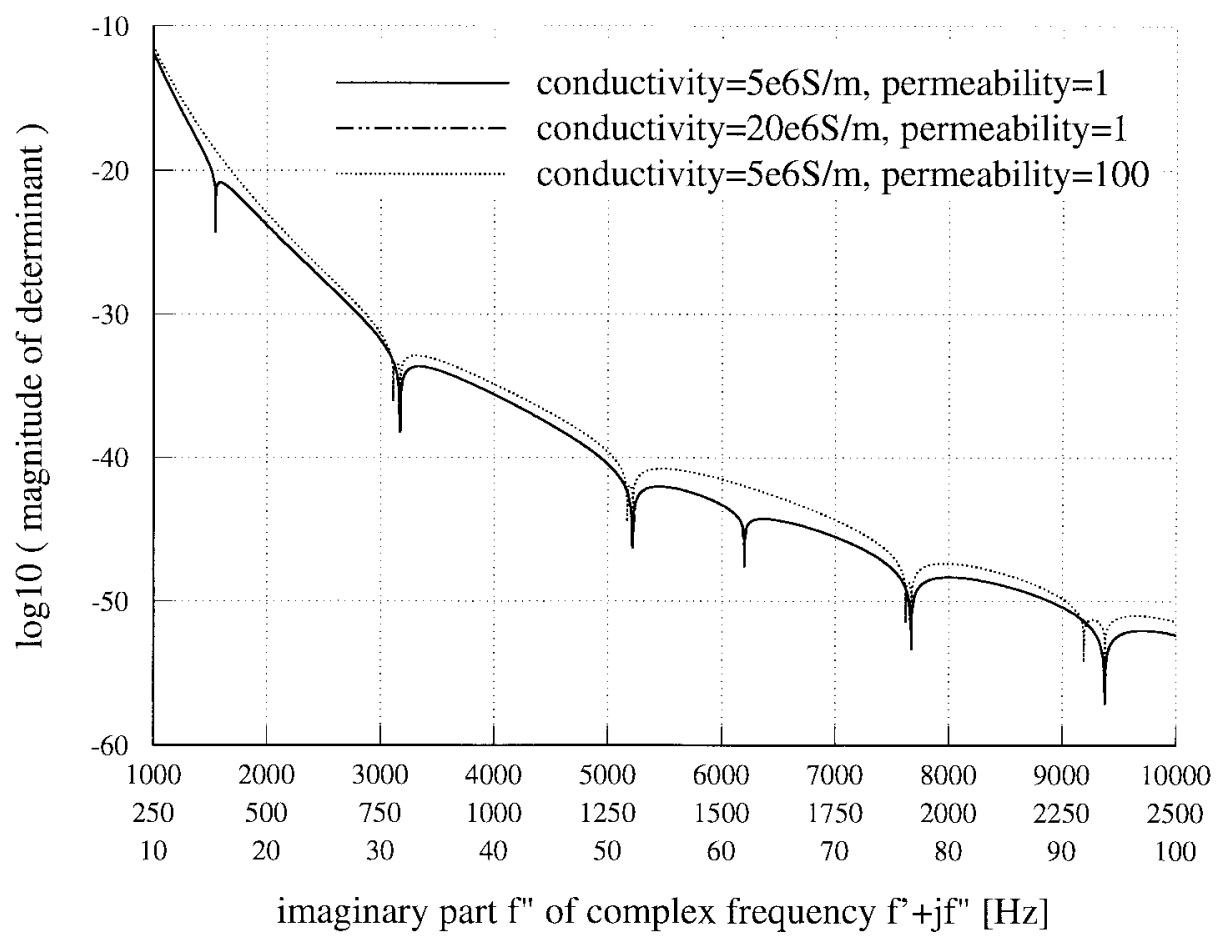

Fig. 2. Magnitude of MoM impedance matrix determinant $(m=0)$ along the imaginary axis of the complex frequency plane for spheres $(a=1.27$ $\mathrm{cm})$ of various material properties.

TABLE I

Comparison Between Measured Decay Time $\tau_{\text {meas }}=1 /\left(2 \pi \cdot \operatorname{Im}\left\{f_{\text {meas }}\right\}\right)[31]-[32]$ AND MoM SoluTION FOR CONDUCTING SPHERES OF RADIUS $a=1.27 \mathrm{~cm}$

\begin{tabular}{l|c|c|c:c}
\hline metal type & $\begin{array}{c}\tau_{\text {meas }} \\
{[\mathrm{msec}]}\end{array}$ & $\begin{array}{c}\operatorname{Im}\left\{f_{\text {meas }}\right\} \\
{[\mathrm{Hz}]}\end{array}$ & $\begin{array}{c}\operatorname{Im}\left[f_{0,1}\right\} \\
{[\mathrm{Hz}]}\end{array}$ & $\begin{array}{c}\operatorname{Re}\left\{f_{0.1}\right\} \\
{[\mathrm{Hz}]}\end{array}$ \\
\hline aluminum $\left(\sigma \approx 30 \cdot 10^{6} \mathrm{~S} / \mathrm{m}\right)$ & $\approx 0.477$ & $\approx 333$ & 260.5 & 0.016 \\
\hline brass $\left(\sigma \approx 12 \cdot 10^{6} \mathrm{~S} / \mathrm{m}\right)$ & $\approx 0.255$ & $\approx 624$ & 651.2 & 0.041 \\
\hline lead $\left(\sigma \approx 5 \cdot 10^{6} \mathrm{~S} / \mathrm{m}\right)$ & $\approx 0.095$ & $\approx 1675$ & 1562.9 & 0.097 \\
\hline stainiess steel $304 \mathrm{SS}\left(\sigma \approx 1.3 \cdot 10^{6} \mathrm{~S} / \mathrm{m}\right)$ & $\approx 0.026$ & $\approx 6121$ & 6011.1 & 0.374 \\
\hline
\end{tabular}

TABLE II

Comparison Between Measured Decay Time $\tau_{\text {meas }}=1 /\left(2 \pi \cdot \operatorname{Im}\left\{f_{\text {meas }}\right\}\right)$ [31]-[32] AND MoM SOLUTION FOR Aluminum Disks of RADIUS $a=2.5 \mathrm{~cm}$ AND DifFERENT THICKNESSES

\begin{tabular}{l|c|c|c|c}
\hline $\begin{array}{l}\text { thickness of aluminum disk } \\
\left.\text { (radius } a=2.5 \mathrm{~cm}, \sigma \approx 21 \cdot 10^{6} \mathrm{~S} / \mathrm{m}\right)\end{array}$ & $\begin{array}{c}\tau_{\text {meas }} \\
{[\mathrm{msec}]}\end{array}$ & $\begin{array}{c}\operatorname{Im}\left\{f_{\text {meas }}\right\} \\
{[\mathrm{Hz}]}\end{array}$ & $\begin{array}{c}\operatorname{Im}\left[f_{0.1}\right\} \\
{[\mathrm{Hz}]}\end{array}$ & $\begin{array}{c}\operatorname{Re}\left[f_{0.1}\right\} \\
{[\mathrm{Hz}]}\end{array}$ \\
\hline$h=3.175 \mathrm{~mm}(1 / 8$ inch $)$ & $\approx 0.363$ & $\approx 438$ & 466.1 & 0.169 \\
\hline$h \approx 1.588 \mathrm{~mm}(1 / 16 \mathrm{inch})$ & $\approx 0.164$ & $\approx 970$ & 883.4 & 0.490 \\
\hline$h=0.794 \mathrm{~mm}(1 / 32 \mathrm{inch})$ & $\approx 0.089$ & $\approx 1788$ & 1718.3 & 1.213 \\
\hline$h \approx 0.508 \mathrm{~mm}(1 / 50 \mathrm{inch})$ & $\approx 0.058$ & $\approx 2744$ & 2658.7 & 1.483 \\
\hline
\end{tabular}

times are also available in [31]-[32] (Table II). Although the disks were machined from different sheets of aluminum of different alloys and unknown conductivities [32], we have assumed the same conductivity of $\sigma=21 \cdot 10^{6} \mathrm{~S} / \mathrm{m}$ for all disks. The fact that the decay time scales approximately with thickness does not violate (20) because (20) was derived assuming that the target shape does not change. Here only the thickness varies while the radius $a$ is constant.

The resonant surface currents, i.e., the nontrivial solution of (17) for zero driving vector $V^{m}=0$ at a given resonant frequency, can be used for mode identification. For the aluminum disk with $h \approx 3.175 \mathrm{~mm}$ (1/8 in.) Fig. 3 shows the resonant surface current distribution for the two lowest modes. Only the $\phi$-component of the electric and the $t$-component of the magnetic surface currents are nonzero $\left(\mathrm{TE}^{z}\right.$-modes with $m=0$ ). That is, because at very low frequencies, a thin disk can only support currents in circular paths around the axis of symmetry.

\section{Cylinder}

Despite the fact that a circular cylinder is very simple in shape, there is no closed form solution available for its natural resonances or scattering behavior. We discuss an approximate solution for modes with $m=0$ later, but let us first compare numerical results with measured decay times for a solid cylinder of radius $a=1.27 \mathrm{~cm}$, height $h=2.54 \mathrm{~cm}$, and different conductivities. The decay times derived from the measured step responses in [31]-[32] and converted into imaginary resonant frequencies are compared to the calculated ones (Table III). The slight discrepancies could be due to the fact that the true conductivities of the targets are unknown.

Fig. 4 shows the frequency-dependent magnitude of the MoM determinant for different material properties, using different scales on the imaginary frequency axes. Again, all resonant frequencies scale almost exactly with $1 / \sigma$ (the two curves for nonpermeable cylinders lie on top of each other and so only one curve is visible), but only a few resonances scale with $1 / \mu$. Note also that the resonant frequencies for highly permeable objects occur in pairs with only slightly shifted resonant frequencies. We now give a simple explanation for 


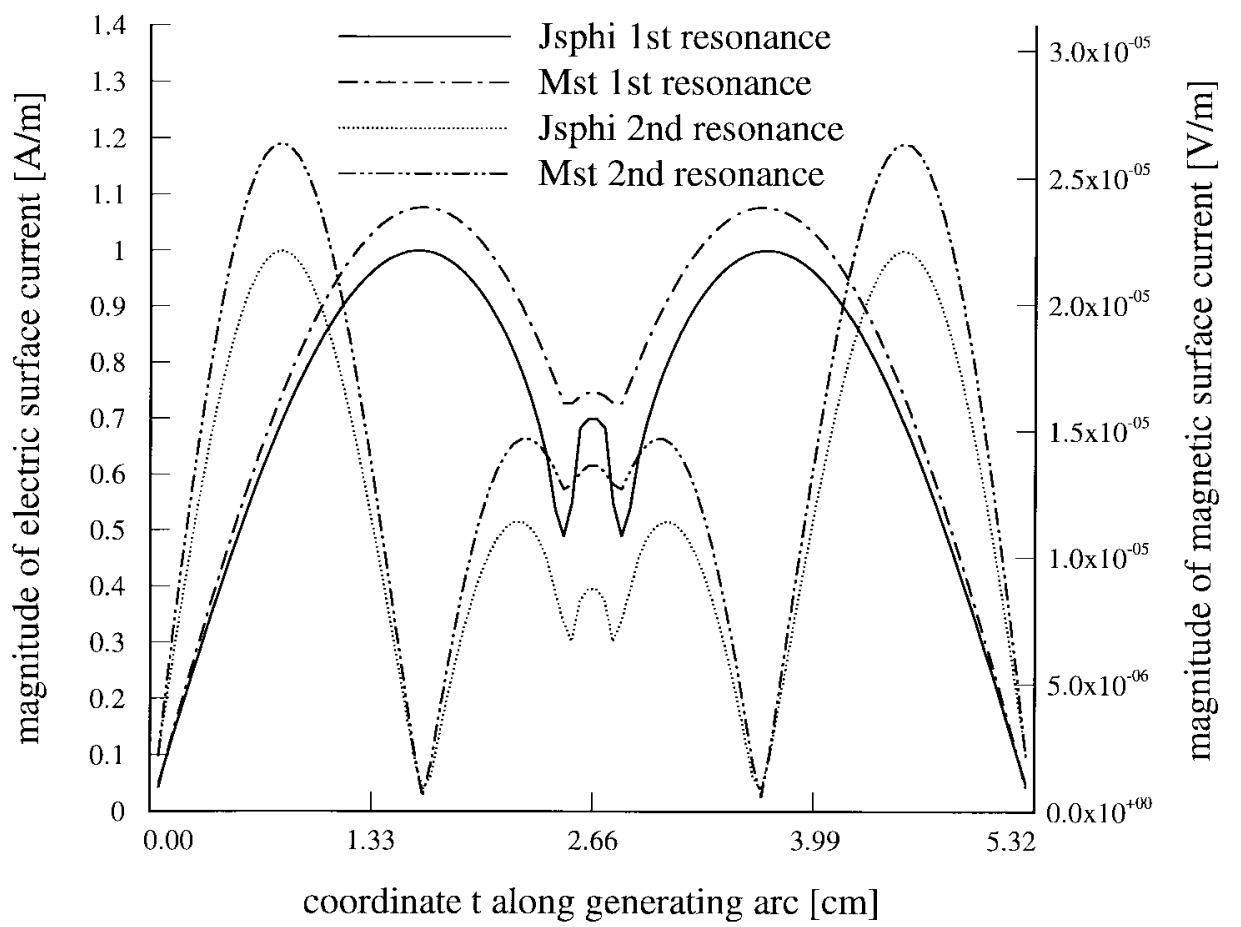

Fig. 3. Electric and magnetic resonant surface currents for the lowest two resonances $\left(m=0\right.$, TE ${ }^{z}$-modes $)$ of a 5-cm diameter aluminum disk with thickness $h \approx 3.175 \mathrm{~mm}$ (1/8 in.).

TABLE III

Comparison Between Measured Decay Time $\tau_{\text {meas }}=1 /\left(2 \pi \cdot \operatorname{Im}\left\{f_{\text {meas }}\right\}\right)[31]-[32]$ AND MoM SOLUTION FOR Aluminum Disks of RAdius $a=1.27 \mathrm{~cm}$ AND HeIGHT $h=2.54 \mathrm{~cm}$

\begin{tabular}{l|c|c|c|c}
\hline metal typc & $\begin{array}{c}\tau_{\text {mecas }} \\
{[\mathrm{msec}]}\end{array}$ & $\begin{array}{c}\operatorname{Im}\left[f_{\text {macas }}\right\} \\
{[\mathrm{Hz}]}\end{array}$ & $\begin{array}{c}\operatorname{Im}\left\{f_{0,1}\right\} \\
{[\mathrm{Hz}]}\end{array}$ & $\begin{array}{c}\operatorname{Re}\left\{f_{0,1}\right\} \\
{[\mathrm{Hz}]}\end{array}$ \\
\hline aluminum $\left(\sigma \approx 30 \cdot 10^{6} \mathrm{~S} / \mathrm{m}\right)$ & $\approx 0.620$ & $\approx 257$ & 208.6 & 0.006 \\
\hline brass $\left(\sigma \approx 12 \cdot 10^{6} \mathrm{~S} / \mathrm{m}\right)$ & $\approx 0.295$ & $\approx 539$ & 521.7 & 0.014 \\
\hline lead $\left(\sigma \approx 5 \cdot 10^{6} \mathrm{~S} / \mathrm{m}\right)$ & $\approx 0.096$ & $\approx 16.58$ & 1251.9 & 0.034 \\
\hline stainless stecl $304 \mathrm{SS}\left(\sigma \approx 1.3 \cdot 10^{6} \mathrm{~S} / \mathrm{m}\right)$ & $\approx 0.036$ & $\approx 4421$ & 4815.4 & 0.130 \\
\hline
\end{tabular}

this behavior. Neglecting displacement currents $(\sigma \gg \omega \varepsilon)$ and assuming a real permeability, the wavenumber inside the target is real for purely imaginary frequencies [see (19)]. Therefore, we can apply the theory for circular cross-section cavities [37], if we modify the boundary conditions to derive the radial wavenumber $k_{p}$. For $\mathrm{TM}_{0 n p}^{z}$-modes (azimuthal mode index $m=0$, radial mode index $n$, axial mode index $p$ ), i.e., modes with the magnetic field only in the $\phi$-direction, the boundary condition for the normal component of the electric field at the surface can be satisfied only if $E_{p}(\rho=a) \approx 0$ and $E_{z}(z= \pm h / 2) \approx 0$ because $\sigma+\omega \varepsilon \approx \sigma \gg \omega \varepsilon_{0}$. The resonant frequencies are therefore given by

$$
\begin{aligned}
& f_{0 n p}^{\prime \prime} \approx \frac{1}{2 \pi \sigma \mu} {\left[\left(\frac{\chi_{0 n}^{\prime}}{a}\right)^{2}+\left(\frac{p \pi}{h}\right)^{2}\right] \text { with } } \\
& n=1,2,3, \cdots \text { and } p=1,2,3, \cdots
\end{aligned}
$$

where $\chi_{0 n}^{\prime}$ represents the $n$th zero of the derivative of the Bessel function $J_{0}(x)$. For nonpermeable targets, the corresponding $\mathrm{TE}^{z}$-modes have lower resonant frequencies because the magnetic field components $H_{\rho}$ and $H_{z}$ inside the target have finite values at the boundary. For increasing permeability, however, the resonant frequencies of the $\mathrm{TE}^{z}$-modes converge to those of the $\mathrm{TM}_{0 n p^{2}}^{z}$-modes given in (31) (pairs of resonances in Fig. 4) because, dual to the $\mathrm{TM}^{z}$-case, the boundary conditions $H_{\rho}(\rho=a) \approx 0$ and $H_{z}(z= \pm h / 2) \approx 0$ have to be satisfied for $\mu \gg \mu_{0}$. The numerically calculated resonant frequencies for $\mathrm{TM}_{\mathrm{O} m p}^{z}$-modes and the theoretical ones in (31) agree within less than $0.02 \%$ for all modes included in Fig. 4 ( $m n p=011,012,013,014,021,022)$, providing an excellent verification of our MoM code for BOR type objects.

To illustrate the explanation above, Figs. 5 and 6 show the resonant surface currents of corresponding $\mathrm{TE}^{z}$ - and $\mathrm{TM}^{z}$-modes (for $m=0$ ) for a nonpermeable cylinder with conductivity $\sigma=5 \cdot 10^{6} \mathrm{~S} / \mathrm{m}$ and the same dimensions as in Table III. The principal behavior of the electric/magnetic surface currents for the $\mathrm{TE}^{z}$-modes is about the same as the magnetic/electric surface currents for the $\mathrm{TM}^{z}$-modes. However, while the $M_{s \phi}$-currents (corresponding to $E_{t}$ at the surface) for the $\mathrm{TM}^{z}$-modes vanish at the cylinder edges, this is not true for the $J_{s \phi}$-currents (corresponding to $H_{t}$ at the surface). Therefore, for $\mathrm{TE}^{z}$-modes, the normal magnetic field does not vanish at the interface between target and free space. Increasing the permeability to $\mu_{r}=100$, the resonant frequencies of these two $\mathrm{TE}^{z}$-modes $(j 26.24$ and $j 57.52 \mathrm{~Hz})$ approach those of the $\mathrm{TM}^{z}$-modes $(j 26.94$ and $j 57.94 \mathrm{~Hz})$, where the latter ones almost exactly scale with $1 / \mu$. The $\mathrm{TM}^{z}$ resonant surface currents for the permeable cylinder look exactly like those in Fig. 6 (the electric surface currents only scaled by a factor $1 / \mu_{r}$ ) and the $\mathrm{TE}^{z}$ surface currents show an almost dual behavior with no obvious differences $\left(J_{s \phi}\right.$ looks like $M_{s \phi}$ in Fig. 6, $M_{s t}$ only scaled by a factor of approximately 1400 compared to $J_{s t}$ in Fig. 6). 


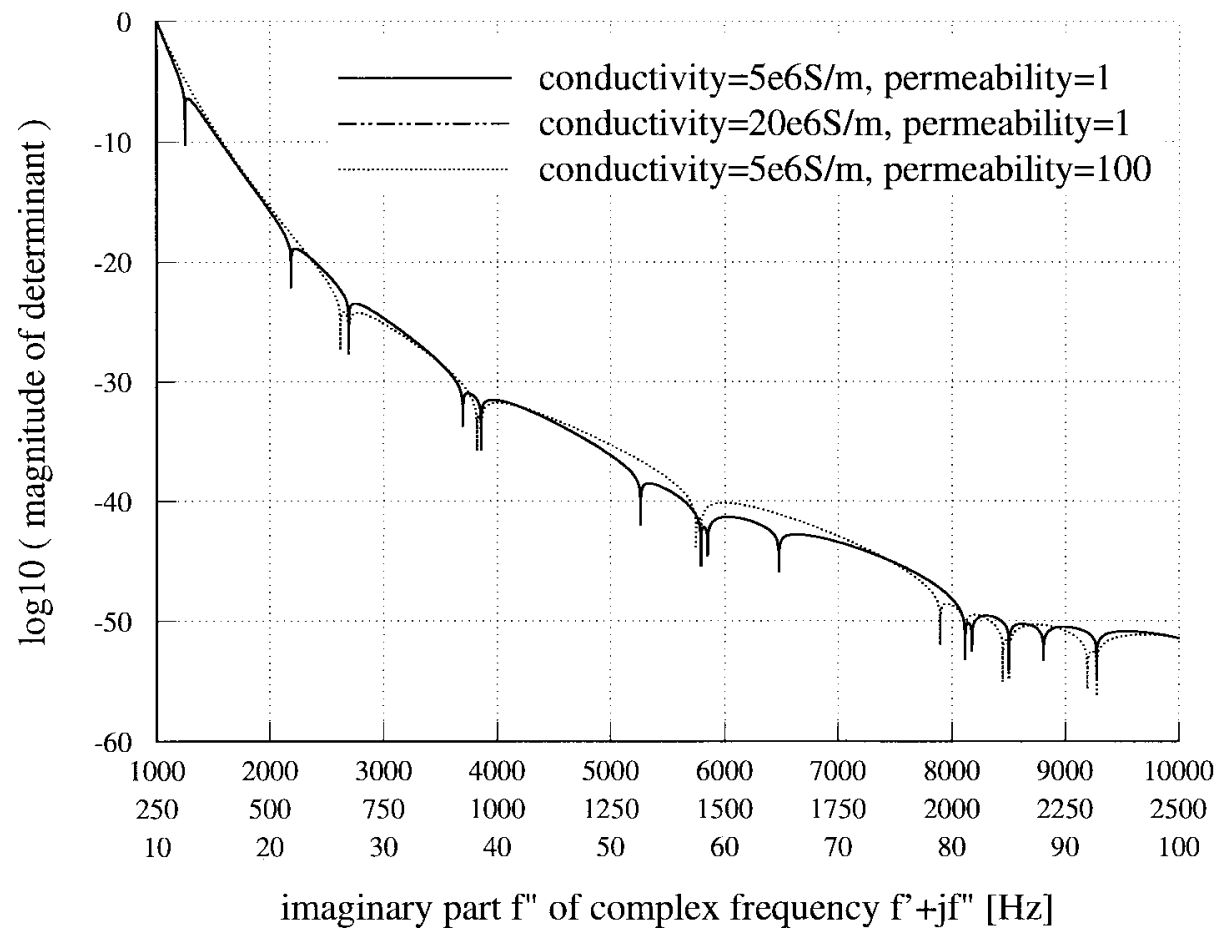

Fig. 4. Magnitude of MoM impedance matrix determinant $(m=0)$ along the imaginary axis of the complex frequency plane for cylinders $(a=1.27$ $\mathrm{cm}, h=2.54 \mathrm{~cm}$ ) of various material properties.

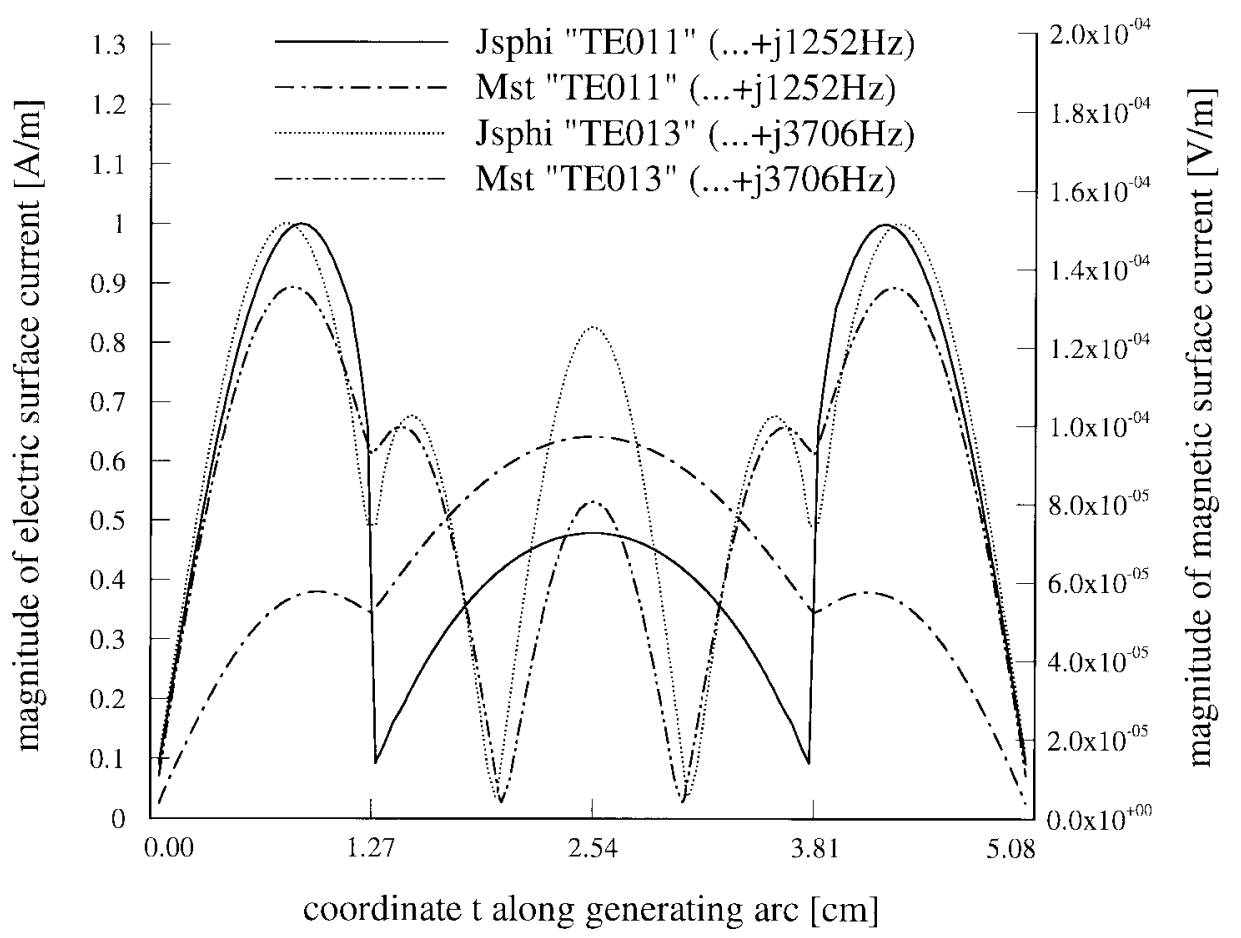

Fig. 5. Electric and magnetic resonant surface currents for two different $\mathrm{TE}^{z}$-modes $(m=0)$ of a conducting and nonpermeable cylinder $\left(\sigma=5 \cdot 10^{6}\right.$ $\mathrm{S} / \mathrm{m}, a=1.27 \mathrm{~cm}, h=2.54 \mathrm{~cm}$ ).

\section{BOR of Arbitrary Shape}

The MoM solution in Section II-B is applicable not only to canonical type targets (sphere, disk, cylinder) but to arbitrary BOR's. As an example, we now look at the natural resonances of the target shown as inset in Fig. 7, which we believe cannot be calculated analytically. The characteristic dimension of the target is about the same as for the sphere and the cylinder in Section IV-A and C, respectively, and therefore we expect resonant frequencies of the same order. For the first three $\mathrm{TE}^{z}$ - and $\mathrm{TM}^{z}$-modes with $m=0$ the calculated resonant frequencies are given in Table IV for the nonpermeable conducting case $\left(\sigma=5 \cdot 10^{6} \mathrm{~S} / \mathrm{m}\right)$ as well as for conductivity 


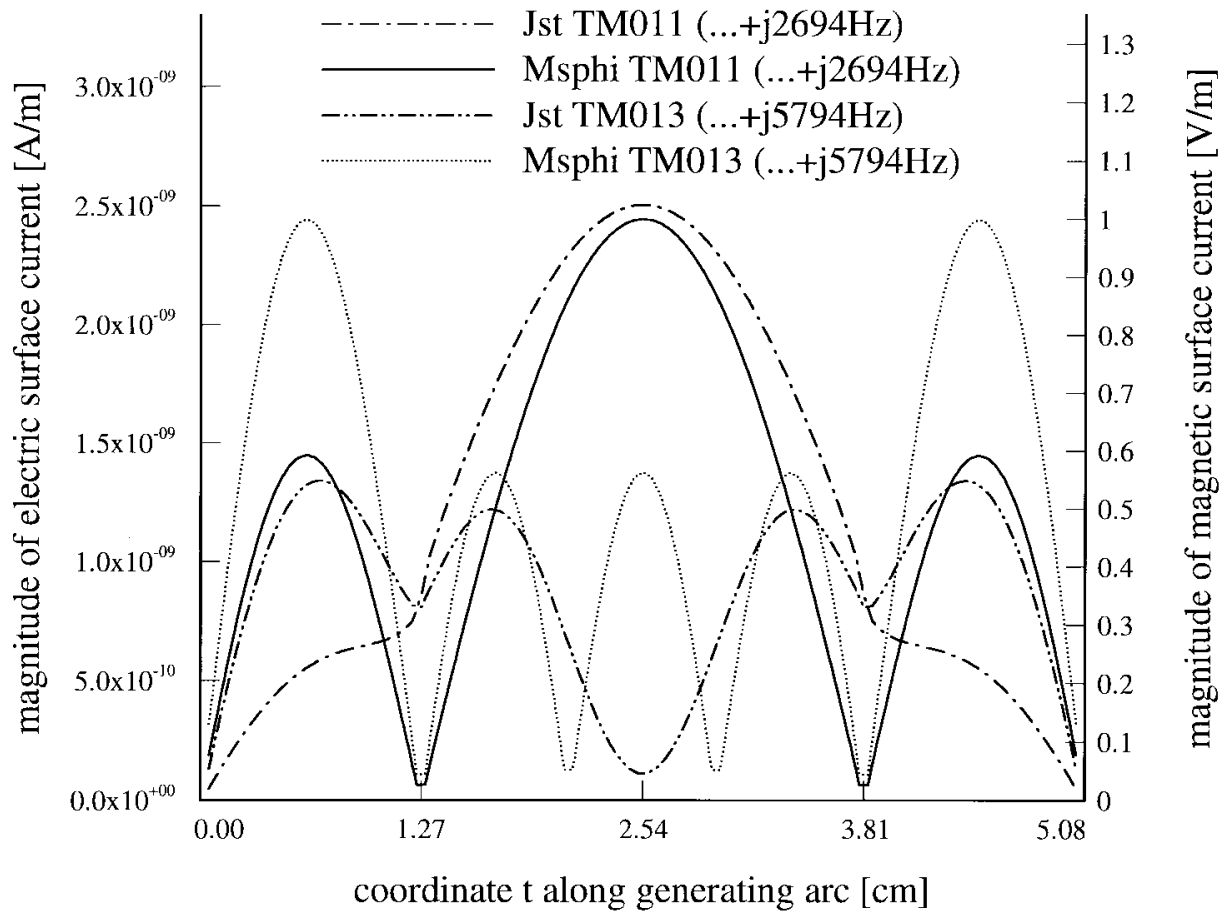

Fig. 6. Electric and magnetic resonant surface currents for two different modes of a conducting and nonpermeable cylinder $\left(\sigma=5 \cdot 10^{6} \mathrm{~S} / \mathrm{m}\right.$, $a=1.27 \mathrm{~cm}, h=2.54 \mathrm{~cm}$ ).

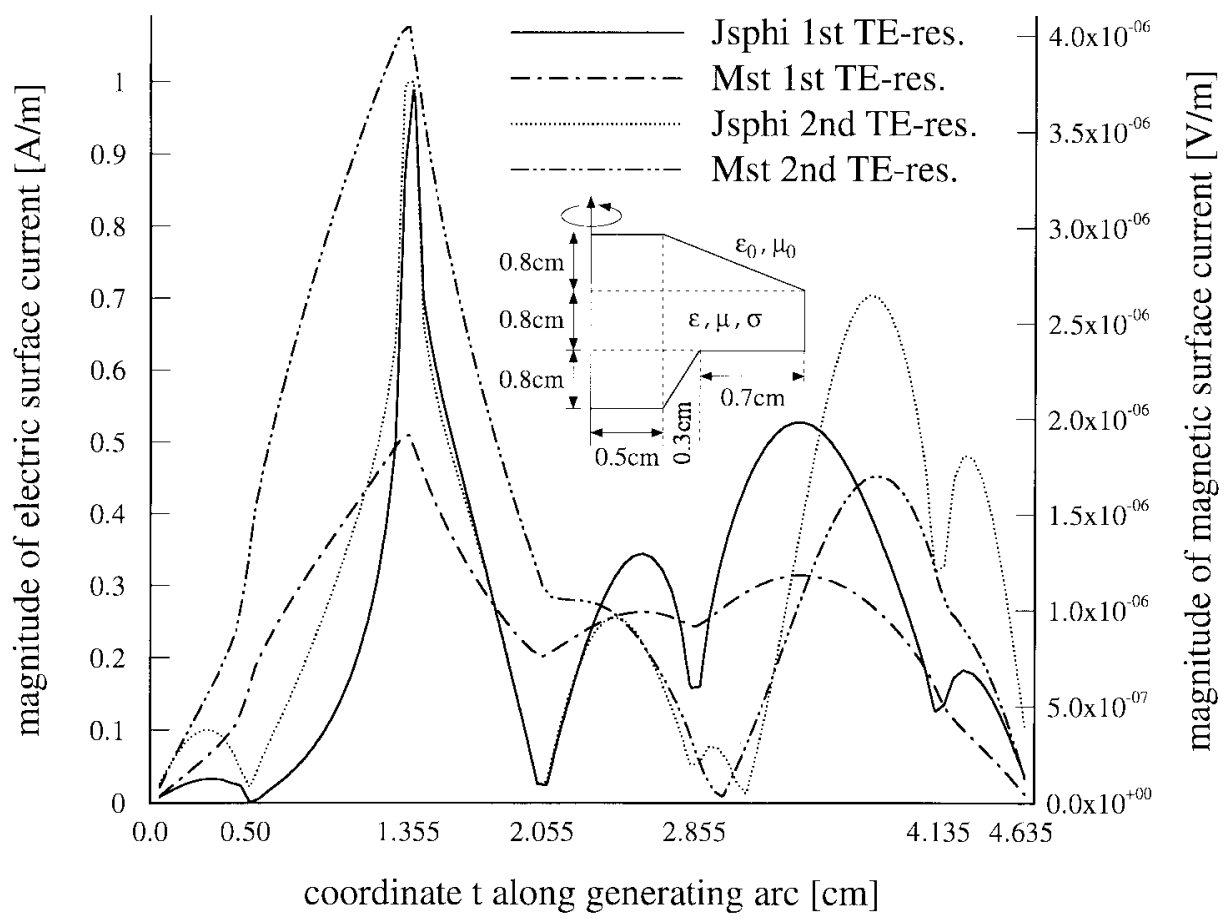

Fig. 7. Electric and magnetic resonant surface currents for the first two $\mathrm{TE}^{z}$ modes $(m=0)$ of a target (shown as inset) with conductivity $\sigma=5 \cdot 10^{6}$ $\mathrm{S} / \mathrm{m}$ and relative permeability $\mu_{r}=100$.

$\sigma=5 \cdot 10^{6} \mathrm{~S} / \mathrm{m}$ and relative permeability $\mu_{r}=100$. As for the canonical objects before, the resonant frequencies for the $\mathrm{TM}^{z}$-modes exactly scale with $1 / \mu$ (not the case for the $\mathrm{TE}^{z}$-modes), and the $\mathrm{TE}^{z}$ resonant frequencies approach those for the $\mathrm{TM}^{z}$-modes for increasing permeabilities. Finally, Fig. 7 shows the resonant surface currents for the lowest two
$\mathrm{TE}^{z}$-modes for the permeable case in Table IV $\left(\sigma=5 \cdot 10^{6}\right.$ $\left.\mathrm{S} / \mathrm{m}, \mu_{r}=100\right)$.

\section{E. Example for $C R L B$}

Let us consider as an example the CRLB (Section III) for the cylinder of radius $1.27 \mathrm{~cm}$ and height $2.54 \mathrm{~cm}$ in 


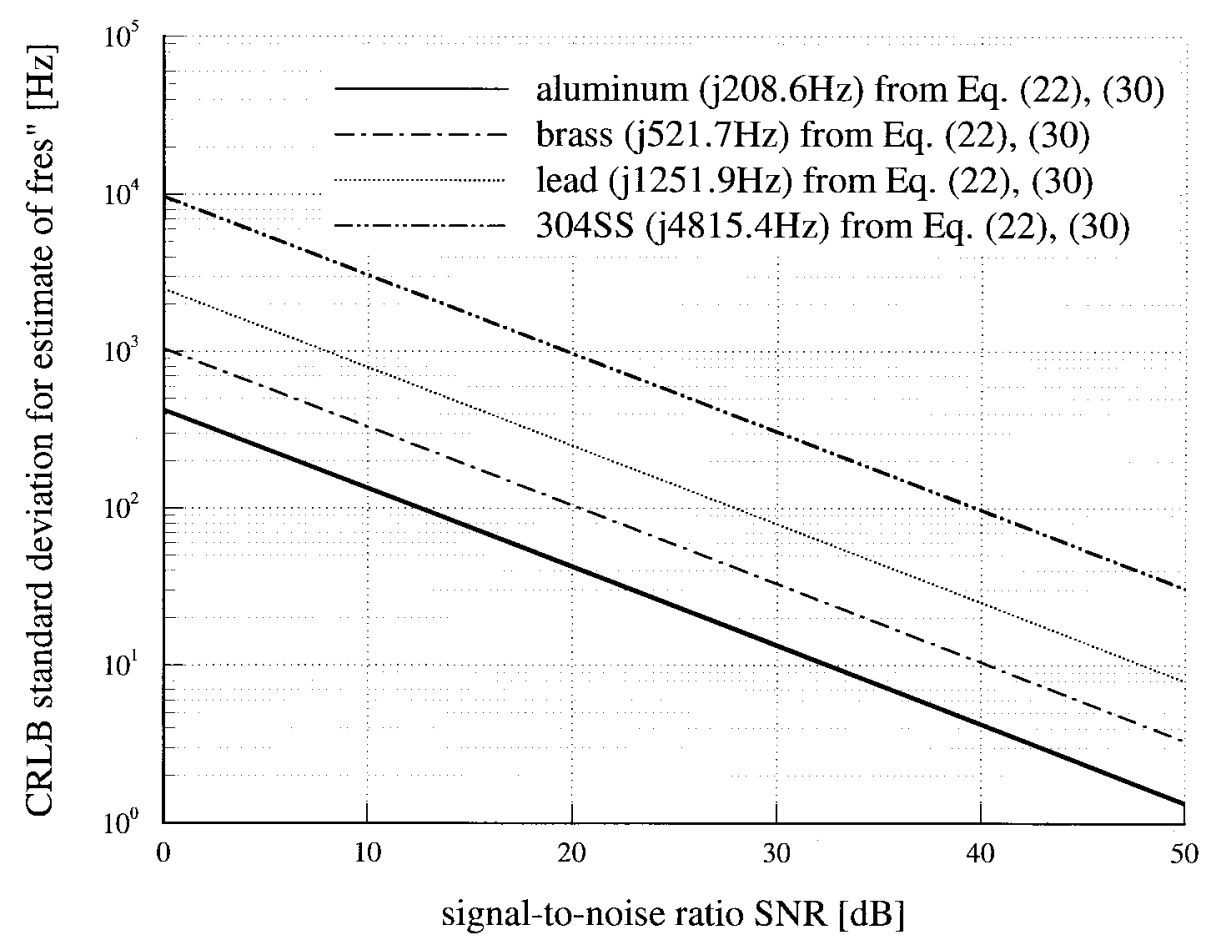

Fig. 8. Minimum standard deviation (Cramer-Rao) for the estimate of the imaginary part of the complex resonant frequency of conducting cylinders made of metals with different conductivities $(a=1.27 \mathrm{~cm}, h=2.54 \mathrm{~cm})$ for sampling with $N=256$ and $\Delta t=10 \mu \mathrm{s}$.

TABLE IV

RESONANT FREQUencies OF THE TARget SHOWn IN THE INSET OF Fig. 7 FOR $m=0$ AND DifFERENT PERMEABILITIES

\begin{tabular}{|c|c|c|c|}
\hline \multicolumn{2}{|c|}{$\sigma=5 \cdot 10^{6} \mathrm{~s} / \mathrm{m}$ and $\mu_{1}=1$} & \multicolumn{2}{|c|}{$\sigma=5 \cdot 10^{6} \mathrm{~s} / \mathrm{m}$ and $\mu_{r}=100$} \\
\hline$f_{0, v}\left[\mathrm{~Hz} \mid \mathrm{TL}^{2}\right.$-modes & $f_{0, v}[\mathrm{~Hz}] \mathrm{TM}^{z}$-modes & $f_{0, v}[\mathrm{~Hz}] \mathrm{TE}^{2}$-modes & $f_{0 . v}[\mathrm{~Hz}] \mathrm{TM}^{2}$-modes \\
\hline $0.124+\mathrm{j} \cdot 1370.8$ & $0.035+\mathrm{j} \cdot 3170.9$ & $0.00036+j \cdot 31.007$ & $0.00035+j \cdot 31.709$ \\
\hline $0.262+\mathrm{j} \cdot 3638.4$ & $0.132+\mathrm{j} \cdot 6518.3$ & $0.00124+j .64 .083$ & $0.00132+j .65 .183$ \\
\hline $0.714+\mathrm{j} \cdot 4489.3$ & $0.145+\mathrm{j} .7008 .5$ & $0.00170+\mathrm{j} \cdot 69.336$ & $0.00145+\mathrm{j} \cdot 70.085$ \\
\hline
\end{tabular}

Section IV-C. The lowest resonant frequency, corresponding to the longest decay time, is $j 208.6 \mathrm{~Hz}$ for aluminum, $j 521.7 \mathrm{~Hz}$ for brass, $j 1251.9 \mathrm{~Hz}$ for lead, and $j 4815.4 \mathrm{~Hz}$ for stainless steel, respectively (see Table III). Taking into account only this lowest resonance, the standard deviation for the imaginary part of the complex resonant frequency as a function of the SNR is given in Fig. 8. Since we used $\Delta t=10 \mu \mathrm{s}$ and $N=256$ here (conditions in (29) are satisfied), the CRLB results from (30) differ only slightly from the general solution in (22) (the curves lie on top of each other in Fig. 8). Note that (30) gives the CRLB for the relative variance, but the standard deviation is plotted in Fig. 8. Moreover, if we assume $\Delta$ represents the minimum difference between the resonant frequencies considered above, and that a standard deviation of $0.25 \Delta$ is required for unambiguous identification of the four targets, an SNR of about $23 \mathrm{~dB}$ is needed in this example. If we relax this condition to $0.5 \Delta$, an SNR of $17 \mathrm{~dB}$ is sufficient.

The example above considered a fixed target shape with variable conductivities. Now we vary the shape of a target while the conductivity and permeability are fixed. We are interested in the minimum SNR required to distinguish between $5-\mathrm{cm}$ diameter aluminum disks of different thickness $h\left(\sigma=21 \cdot 10^{6} \mathrm{~S} / \mathrm{m}, \mu=\mu_{0}\right)$ (see Section IV-B). The MoM results for the lowest resonant frequencies (longest decay times) for $h \approx 3.175,1.588,0.794$, and $0.508 \mathrm{~mm}$ $(1 / 8,1 / 16,1 / 32$, and $1 / 50$ in) can be found in Table II. The minimum standard deviation for the imaginary part of the complex resonant frequency (CRLB) as a function of SNR is given in Fig. 9 for the four different thicknesses. Although the coarse sampling assumed here $(\Delta t=50 \mu \mathrm{s}, N=16)$ does not satisfy the conditions in (29) very well, the error using (30) is still small [see Fig. 9, the true standard deviations from (22) are slightly larger than those from (30)]. If we again assume that a standard deviation less than $0.25 \Delta$ (definition of $\Delta$ analogue to first example) is required for unambiguous identification of the disks, an SNR of about $28 \mathrm{~dB}$ is needed (for $0.5 \Delta$, an SNR of $22 \mathrm{~dB}$ is sufficient). In like manner, SNR requirements can be computed for any set of targets of interest.

\section{CONCLUSIONS}

A circuit-based analysis of low-frequency, electromagnetic scattering from conducting targets has been presented, from which it was demonstrated that the late-time (natural) response from such targets is represented by an exponentially damped response. A rigorous, MoM analysis was then applied to the case of conducting, permeable BOR's in free space, for which the damped natural response was demonstrated as still appropriate. Moreover, the MoM-computed decay constants were found to be in good agreement with measured data.

Several important relationships were derived and demonstrated, as follows.

1) For a conducting, nonpermeable target, the modal decay constants were shown to scale with conductivity, and 


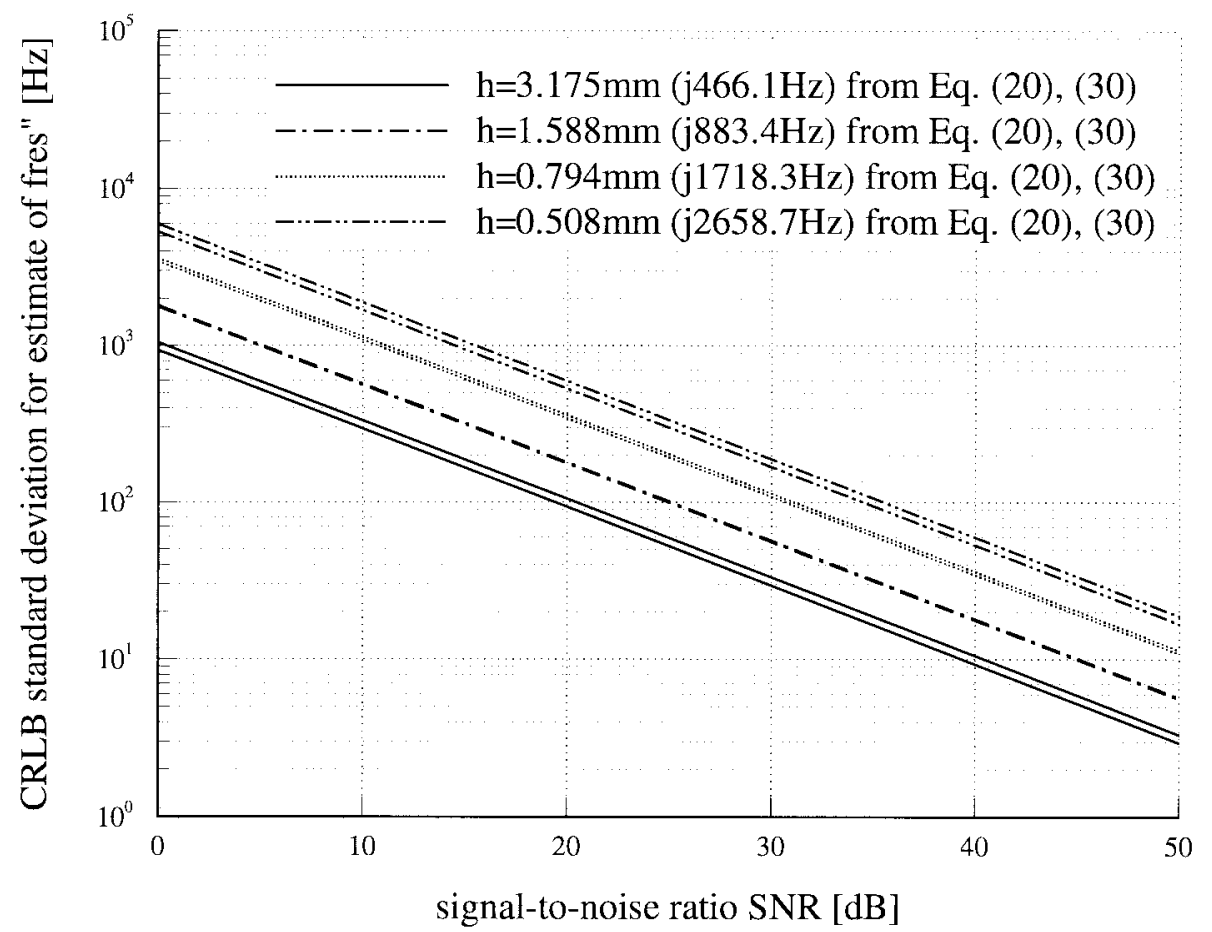

Fig. 9. Minimum standard deviation (Cramer-Rao) for the estimate of the imaginary part of the complex resonant frequency of 5-cm diameter aluminum disks of different thickness $h\left(\sigma=21 \cdot 10^{6} \mathrm{~S} / \mathrm{m}, \mu=\mu_{0}\right)$ for sampling with $N=16$ and $\Delta t=50 \mu$ s [the results from (22) are slightly larger than those using the approximation (30)].

therefore results need only be computed for a representative conductivity, assuming the target shape remains constant.

2) Decay constants (with $m=0$ ) for highly permeable and conducting targets can be computed very accurately ( $<1 \%$ error) using a simple resonant-cavity analysis (particularly useful for simple shapes such as cylinders and discs).

3) CRLB for estimation of the decay parameters can often be simplified to a simple relationship that only depends on the SNR (computed from the noise variance and the decay coefficient).

These relationships allow us to perform quick "back-of-theenvelope" computations of the system requirements for unambiguous target discrimination via decay-parameter estimation. In this context, for the examples considered here, we have found that an SNR of roughly $20 \mathrm{~dB}$ is required.

The low-frequency, decay-constant discrimination of conducting, permeable targets has many applications. For example, we can use it for concealed-weapon identification [31] as well as for identification of buried metal mines (in which case discrimination mitigates the need to dig up each piece of anthropic metal clutter). With regard to the latter example, the effects of the lossy soil become important as the size of the metal target diminishes (this is especially relevant for plastic mines that possess very small metal content). Therefore, in future work, we will consider the BOR targets buried in a lossy half space. This problem constitutes a significant escalation in complexity, due to the need to compute the half-space dyadic Green's function.

\section{REFERENCES}

[1] B. L. Merchant, P. J. Moser, A. Nagl, and H. Überall, "Complex pole patterns of the scattering amplitude for conducting spheroids and finite-length cylinders," IEEE Trans. Antennas Propagat., vol. 36, pp. 1769-1778, Dec. 1988.

[2] S. R. Vechinski and T. H. Shumpert, "Natural resonances of conducting bodies of revolution," IEEE Trans. Antennas Propagat., vol. 38, pp. 1133-1136, July 1990.

[3] C. E. Baum, "Low-frequency near-field magnetic scattering from highly, but not perfectly, conducting bodies," Phillips Lab., Interaction Note 499, Nov. 1993.

[4] S. Vitebskiy and L. Carin, "Moment-method modeling of short-pulse scattering from and the resonances of a wire buried inside a lossy, dispersive half-space," IEEE Trans. Antennas Propagat., vol. 43, pp. 1303-1312, Nov. 1995.

[5] _ " "Resonances of perfectly conducting wires and bodies of revolution buried in a lossy dispersive half-space," IEEE Trans. Antennas Propagat., vol. 44, pp. 1575-1583, Dec. 1996.

[6] C. E. Baum, N. Geng, and L. Carin, "Integral equations and polarizability for magnetic singularity identification," Phillips Lab., Interaction Note 524, Mar. 1997.

[7] F. M. Tesche, "On the analysis of scattering and antenna problems using the singularity expansion technique," IEEE Trans. Antennas Propagat., vol. AP-21, pp. 53-62, Jan. 1973.

[8] L. Marin, "Natural-mode representation of transient scattered fields," IEEE Trans. Antennas Propagat., vol. 21, pp. 809-818, Nov. 1973.

[9] M. L. Van Blaricum and R. Mittra, "A technique for extracting the poles and residues of a system directly from its transient response," IEEE Trans. Antennas Propagat., vol. AP-23, pp. 777-781, Nov. 1975.

[10] A. J. Poggio, M. L. Van Blaricum, E. K. Miller, and R. Mittra, "Evaluation of a processing technique for transient data," IEEE Trans. Antennas Propagat., vol. AP-26, pp. 165-173, Jan. 1978.

[11] D. G. Dudley, "Parametric modeling of transient electromagnetic systems," Radio Sci., vol. 14, pp. 387-396, May-June 1979.

[12] C. E. Baum, R. J. Rothwell, K.-M. Chen, and D. P. Nyquist, "The singularity expansion method and its application to target identification," Proc. IEEE, vol. 79, pp. 1481-1492, Sept. 1991.

[13] C. E. Baum, "On the singularity expansion method for the solution of electromagnetic interaction problems," Phillips Lab., Interaction Note 88, Dec. 1971. 
[14] Y. Hua and T. K. Sarkar, "Generalized pencil-of function method for extracting poles of an EM system from its transient response," IEEE Trans. Antennas Propagat., vol. 37, pp. 229-234, Feb. 1989.

[15] Y. Hua and T. K. Sarkar, "Matrix pencil method for estimating parameters of exponentially damped/undamped sinusoids in noise," IEEE Trans. Acoust., Speech, Signal Processing, vol. 38, pp. 814-824, May 1990.

[16] R. W. P. King and C. W. Harrison, "The transmission of electromagnetic waves and pulses into the earth," J. Appl. Phys., vol. 39, pp. 4444-4452, Aug. 1968.

[17] D. L. Moffatt and R. J. Puskar, "A subsurface electromagnetic pulse radar," Geophysics, vol. 41, pp. 506-518, June 1976.

[18] G. S. Smith and W. R. Scott, "A scale model for studying ground penetrating radars," IEEE Trans. Geosci. Remote Sensing, vol. 27, pp. 358-363, July 1989.

[19] C. E. Baum, Ed., Detection and Identification of Visually Obscured Targets. New York: Taylor \& Francis, 1998.

[20] C. Liu and L. C. Shen, "Numerical simulation of subsurface radar for detecting buried pipes," IEEE Trans. Geosci. Remote Sensing, vol. 29, pp. 795-798, Sept. 1991

[21] M. G. Andreasen, "Scattering from bodies of revolution," IEEE Trans. Antennas Propagat., vol. 13, pp. 303-310, Mar. 1965.

[22] J. R. Mautz and R. F. Harrington, "Radiation and scattering from bodies of revolution," Appl. Sci. Res., vol. 20, pp. 405-435, June 1969.

[23] S. D. Gedney and R. Mittra, "The use of the FFT for the efficient solution of the problem of electromagnetic scattering by a body of revolution," IEEE Trans. Antennas Propagat., vol. 38, pp. 313-322, Mar. 1990.

[24] S. Vitebskiy, K. Sturgess, and L. Carin, "Short-pulse plane-wave scattering from buried perfectly conducting bodies of revolution," IEEE Trans. Antennas Propagat., vol. 44, pp. 143-151, Feb. 1996.

[25] T. Wu and L. L. Tsai, "Scattering from arbitrarily-shaped lossy dielectric bodies of revolution," Radio Sci., vol. 12, pp. 709-718, Sept.-Oct. 1977.

[26] J. R. Mautz and R. F. Harrington, "Electromagnetic scattering from a homogeneous material body of revolution," AEÜ, vol. 33, pp. 71-80, Feb. 1979.

[27] A. W. Glisson and D. R. Wilton, "Simple and efficient numerical methods for problems of electromagnetic radiation and scattering from surfaces," IEEE Trans. Antennas Propagat., vol. 28, pp. 593-603, Sept. 1980.

[28] A. W. Glisson, D. Kajfez, and J. James, "Evaluation of modes in dielectric resonators using a surface integral equation formulation," IEEE Trans. Microwave Theory Tech., vol. 31, pp. 1023-1029, Dec. 1983.

[29] A. W. Glisson, "Integral equation techniques," in Dielectric Resonators, D. Kajfez and P. Guillon, Eds. Dedham, MA: Artech House, 1986 pp. 259-325.

[30] D. Kajfez, A. W. Glisson, and J. James, "Computed modal field distributions for isolated dielectric resonators," IEEE Trans. Microwave Theory Tech., vol. 32, pp. 1609-1616, Dec. 1984.

[31] G. D. Sower and S. P. Cave, "Detection and identification of mines from natural magnetic and electromagnetic resonances," in Proc. SPIE, Orlando, FL, vol. 2496, pp. 1015-1024, 1995.

[32] G. D. Sower, "Eddy current resonances of canonical metallic targets-Theory and measurements," EG\&G MSI, Interaction Note, Feb. 1997.

[33] K. Kroschel, Statistische Nachrichtentheorie-Erster Teil_Signalerkennung und Parameterschätzung. Berlin, Germany: Springer-Verlag, 1986 (in German).

[34] S. M. Kay, Fundamentals of Statistical Signal Processing: Estimation Theory. Englewood Cliffs, NJ: Prentice-Hall, 1993.

[35] W. M. Steedly and R. L. Moses, "The Cramer-Rao bound for pole and amplitude coefficient estimates of damped exponential signals in noise," IEEE Trans. Signal Processing, vol. 41, pp. 1305-1318, Mar. 1993.

[36] R. F. Harrington, Field Computation by Moment Methods. New York: Macmillan, 1968

[37] C. A. Balanis, Advanced Engineering Electromagnetics. New York: Wiley, 1989.

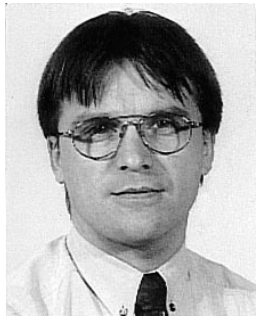

Norbert Geng (S'91-M'96) was born May 14, 1965, in Lauchringen, Germany. He received the Dipl.-Ing. and Dr.-Ing. degrees in electrical engineering from the University of Karlsruhe, Karlsruhe, Germany, in 1991 and 1996, respectively.

$\mathrm{He}$ was with the Institut für Höchstfrequenztechnik und Elektronik (IHE), University of Karlsruhe, from 1991 to 1996, working on full-wave propagation modeling for radio communication systems. In January 1997, he joined the Department of Electrical and Computer Engineering, Duke University, Durham, NC, as a Visiting Postdoctorate for 18 months. His current research interests include computational methods in electromagnetics, with emphasis on short-pulse scattering and wave propagation modeling.

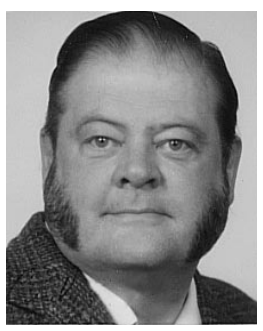

Carl E. Baum (S'62-M'63-SM'78-F'84) was born in Binghamton, NY, on February 6, 1940. He received the B.S. (with honosr), M.S., and Ph.D degrees in electrical engineering from the California Institute of Technology, Pasadena, in 1962, 1963 and 1969 , respectively.

He was commissioned in the United States Air Force in 1962 and was stationed at the Air Force Research Laboratory, Directed Energy Directorate (formerly, Phillips Laboratory, formerly, Air Force Weapons Laboratory), from 1963 to 1967 and from 1968 to 1971. Since 1971, he has served as a civil servant with a position as a Senior Scientist at Phillips Laboratory, Kirtland Air Force Base, Albuquerque, NM. He has published three books: Transient Lens Synthesis: Differential Geometry in Electromagnetic Theory, Electromagnetic Symmetry, and UltraWideband, Short-Pulse Electromagnetics 3. He is Editor of several interagency note series on electromagnetic pulse (EMP) and related subjects. He has led an EMP short course and HPE workshops at numerous locations around the globe.

Dr. Baum received the Air Force Research and Development Award in 1970, the Richard R. Stoddard Award from the IEEE EMC Society in 1984, the IEEE Harry Diamond Memorial Award in 1987 with the citation "for outstanding contributions to the knowledge of transient phenomena in electromagnetics," the AFSC Harold Brown Award in 1990, and Phillips Laboratory Fellow in 1996. He is a member of Commissions A, B, and E of the United States National Committee of the International Union of Radio Science (URSI). He is Founder and President of the SUMMA Foundation, which sponsors various electromagnetics-related activities, including scientific conferences, publications, short courses, fellowships, and awards.

Lawrence Carin (S'85-M'89-SM'96) was born on March 25, 1963, in Washington, DC. He received the B.S., M.S., and Ph.D. degrees in electrical engineering from the University of Maryland, College Park, in 1985, 1986, and 1989 , respectively.

He was with the Electrical Engineering Department at Polytechnic University, Brooklyn, NY, as an Assistant Professor in 1989, and he became an Associate Professor there in 1994. In September 1995, he joined the Electrical Engineering Department, Duke University, Durham, NC, where he is an Associate Professor. His current research interests include quasiplanar transmission lines, short-pulse scattering and propagation, and signal processing.

Dr. Carin is a member of the Tau Beta Pi and Eta Kappa Nu honor societies. 